\title{
La religión y su efecto motivante en el aula L2
}

\section{Religion and Its Motivating Effect on the L2 Classroom}

\author{
Jeremy W. Bachelor ${ }^{1}$
}

\section{Resumen}

Algunos educadores de L2 afirman que la motivación influye de manera importante en el aprendizaje de los estudiantes y lleva a la adquisición exitosa de una lengua extranjera. Como resultado de ello, el estudio de la motivación en los estudiantes L2 es crucial, ya que permite a los investigadores explorar las razones para el éxito (o el fracaso) en el aprendizaje de una lengua extranjera. También permite una mayor comprensión de las formas en que los diferentes tipos de motivación pueden ayudar a los profesores y estudiantes en la dinamización del proceso de aprendizaje de una lengua, lo que conduciría a un mayor éxito del proceso pedagógico. El presente estudio sugiere que la religión es un factor motivante para muchos estudiantes. Al incorporar los ideales religiosos y al hacer conexiones con los textos sagrados en el aula de lengua extranjera se beneficia a los estudiantes que tienen creencias religiosas. Teniendo estos conceptos en mente, es posible diseñar un plan de estudios L2 con los estándares religiosos que también implementan las 5 c de la enseñanza L2. Los profesores pueden involucrar a sus estudiantes en las grandes cuestiones de la identidad cultural y lingüística en última instancia, orientando a los estudiantes hacia una consideración de sus propias identidades. La conclusión es una instrucción L2 más exitosa y estudiantes mejor formados.

Palabras clave

Lengua extranjera, motivación, religión.

\section{Abstract}

There are some L2 educators who say that motivation is the most important factor that influences student learning and the successful acquisition of a foreign language. As a result, a study regarding motivation in L2 students is crucial because it allows researchers to explore reasons for success (or failure) in learning a foreign language. A study of this nature also allows greater insight into the ways that different types of motivation can help teachers and students alike in energizing the process of learning a language, which can bring greater success to the pedagogical process. The present study suggests that religion is a motivating factor for many students. Students in the foreign language classroom who have religious goals may benefit from incorporating religious ideals and making connections with sacred texts. With these concepts in mind, instructors could design an L2 curriculum with religious tenets that implements the "Five Cs" of foreign language instruction. Teachers can engage their students in the larger questions of cultural and linguistic identity, ultimately pointing the students toward a consideration of their own identities. The result is a more successful L2 instruction, in addition to well-rounded students.

\section{Key words}

Foreign language, motivation, religion.

Artículo recibido el 23 de diciembre de 2013 y aprobado el 7 de abril de 2015

1 Heartland Community College, Illinois, Estados Unidos. Correo electrónico: Jeremy.Bachelor@heartland.edu 


\section{Introducción}

Hay muchos factores que contribuyen a la motivación de los estudiantes en el aprendizaje L2 (idioma extranjero) y construcciones teóricas que pretenden explicar estos factores. Un método particularmente útil, sobre todo para aquellos alumnos que muestran el nivel más alto de motivación, tiene que ver con la conexión entre la identidad L2 y su visión. La interacción entre la identidad personal y el idioma es una faceta observable cuando los alumnos proyectan ante sí mismos sus propios futuros como estudiantes de lenguas extranjeras; esta identidad incluye una visión de sí mismos como futuros hablantes de otros idiomas. Como regla general, los investigadores han encontrado en la fe religiosa una fuente de motivación para la gente. Después de todo, es común ver a personas que están motivadas por la devoción o la creencia en una deidad o un conjunto particular de doctrinas religiosas participando de actividades en las que, en otras circunstancias, no participarían. Otra área de los posibles factores de motivación en el aprendizaje de una lengua extranjera tiene que ver con el deseo de leer un texto especialmente significativo. Por ejemplo, un estudiante de literatura podría aprender a leer francés para poder leer a Voltaire en su idioma original, un moralista podría aprender ruso para leer a Dostoievski, o un poeta podría aprender alemán para poder leer a Goethe. A los efectos de esta investigación, el texto es la Biblia, el libro sagrado de la cristiandad; aunque se podría sustituir en este análisis por otro texto sagrado que corresponda según la religión en cuestión. Los datos de estas observaciones casan bien con las investigaciones ya existentes, que sugieren que los alumnos L2 muestran mayor motivación cuando la instrucción se lleva a cabo con materiales auténticos que se relacionan directamente con sus necesidades mundanas lingüísticas (Gilmore, 2007).

Es cierto que existen otras fuentes de motivación para el aprendizaje de las lenguas que no tienen nada que ver con los textos sagrados ni con la religión. Miller y Delaney (2005) señalan que lo religioso ha sido un tema considerado fuera de los límites en el campo de la psicología en su conjunto, mientras que
Maehr (2005) señala que los estudios psicológicos de la motivación han pasado por alto, en gran medida, la importancia que las creencias religiosas tienen sobre las acciones en la formación, los pensamientos y las emociones; observa que la religión "a menudo influye en ciertos procesos psicológicos básicos [...] $y$ ha sido, y sigue siendo, una poderosa fuerza motivadora en la vida de muchas personas ${ }^{2 "}$ (p. 141). En relación con esta observación, varios investigadores han ampliado la sinergia entre la motivación, la identidad personal y el aprendizaje de lenguas para incluir cuestiones de fe. Joseph (2004), por ejemplo, en su metodología, analiza una identidad espiritual que incluye la fuente más profunda para comprender el propósito y el significado de la vida y la existencia misma del cosmos: "las identidades étnicas y religiosas [...] suministran la trama de las historias de nuestras vidas, por separado y colectivamente, y están ligadas a nuestras creencias más profundas sobre la vida, el universo y todo ${ }^{3 \prime \prime}$ (p. 172).

La globalización y los recientes problemas que afectan al mundo han hecho que las cuestiones de fe religiosa surjan en conversaciones diferentes como la lucha contra el terrorismo y otros conflictos internacionales (Coupland, 2010); Mooney (2010) señala acertadamente que "hoy la comprensión de las fuerzas religiosas es quizás más alta en la agenda de la comunidad internacional que desde hace muchos años" (p. 340). Con esto en mente, es importante tener en cuenta que el idioma siempre ha jugado un papel curioso en la práctica religiosa y, de hecho, una gran parte de la fe se ha filtrado a través de los textos sagrados y el lenguaje. Así se trate de los siglos de los comulgantes romanos católicos, que adoraban a través de una lengua latina que la mayoría no entendía; o de los que en su lugar conectaban con Dios a través de la música e imágenes visuales; los protestantes, cuyo deseo de leer la Biblia inspiró la publicación del primer libro impreso (la Biblia de Gutenberg) para su difusión; o los miembros de la Iglesia de Jesucristo de los Santos de los Últimos

2 La traducción es propia.

3 La traducción es propia.

4 La traducción es propia. 
Días, cuyos textos sagrados han sido objeto de decenas de cambios desde su revelación original en el siglo XIX, la relación entre el creyente y los textos de creencia ha sido muy estrecha. En los recientes debates sobre los dilemas éticos y pedagógicos que enfrentan los instructores de idiomas extranjeros que usan un plan de estudios basado en los valores religiosos, la relación entre el aprendizaje L2 y la fe ha surgido especialmente teniendo en cuenta los vientos cambiantes de la religión en la era global (McGrath, 2010; Thomas, 2005; Mooney, 2010; Wong y Canagarajah, 2009). La conexión entre la fe y la lingüística procede del trabajo seminal de Crystal (1965), que más tarde sería conocido como la "teolingüística" (Mooney, 2010); indudablemente, es un área en la que hay una cantidad considerable de interés.

Los que quieran diseñar programas y currículos exitosos para los cursos L2 deben adoptar todas las formas de proporcionar motivación a los estudiantes. El Consejo Americano para la Enseñanza de Lenguas Extranjeras (1996), o American Council on the Teaching of Foreign Languages (ACTFL), sugiere cinco principios de diseño curricular, conocidos comúnmente como " 5 c": comunicación, culturas, conexiones, comparaciones y comunidades. Encontrar maneras de incorporar las ideas religiosas en el aula L2 puede aportar los beneficios de una mayor motivación por parte de los estudiantes en su rendimiento.

\section{Revisión literaria}

Dada esta estrecha relación entre la fe, la identidad $y$ el lenguaje, vale la pena examinar las formas en que estos factores contribuyen a la motivación en la tarea de aprender idiomas. Es esta conexión la que hace que las posibilidades de motivación de los textos sagrados queden claras, ya que pueden ayudar a los estudiantes a combinar las identidades religiosas y lingüísticas para producir un efecto beneficioso para el aprendizaje. La idea psicológica del "ser posible" (Markus y Nurius, 1986) ha servido como un principio útil para explorar esta relación porque, al igual que Maehr (2005) ha observado, la religión juega un papel importante en la construcción del ideal y el autopotencial. Sobre la base de esta teoría del autopotencial, Dörnyei $(2005,2009)$ ha reconstruido la construcción del yo futuro para trabajar con el contexto de aprendizaje de un L2. Mientras los aprendices imaginan escenarios de sus propios futuros en términos de lo que pueden llegar a ser o temen llegar a ser en relación con el desarrollo de esta nueva identidad lingüística, estos potenciales entes lingüísticos futuros se convierten en los avatares que representan sus sueños y metas. Este paradigma se ha aclarado en una construcción con tres componentes: el modelo L2 motivational self system o sistema motivacional del yo (Lepp-Kaethler y Dörnyei, 2012):

1. Yo ideal: La faceta del yo ideal tiene que ver con el aprendizaje L2: si la persona que uno quiere ser habla un idioma en particular, ese yo ideal sirve como un fuerte motivador por aprender el nuevo lenguaje a causa de un deseo intrínseco para disminuir la diferencia entre el ideal y el real. La imagen misma de ser alguien que puede hablar una L2 motiva a los estudiantes a estudiar y a aprender.

2. Yo debería: Alude a características que uno siente que son necesarias para cumplir con las expectativas de la sociedad y para eludir los posibles resultados negativos derivados de su incumpliendo.

3. Experiencia de aprendizaje: Impacto que se produce cuando la identidad real del alumno L2 entra en contacto con el ambiente de aprendizaje, que incluye el impacto del plan de estudios, el profesor, los compañeros y el contacto con el éxito.

El estudio de la religión y la lingüística ha estado recibiendo cada vez más atención en los últimos decenios (Mooney, 2010). Sin embargo, este estudio no ha llegado a centrarse en la motivación personal y el progreso del alumno, por lo menos no hasta este punto. Deci y Ryan (1985) desarrollaron una teoría de la autodeterminación de la motivación, la cual Bakar, Sulaiman y Rafaai (2010) usaron para estructurar un estudio cuantitativo de la motivación 
de los musulmanes para aprender árabe como lengua extranjera. Además de algunos factores establecidos, como la motivación extrínseca e intrínseca, también discutieron una nueva herramienta llamada "la motivación religiosa" que, según sus resultados, contribuyó a la motivación general que los alumnos sentían cuando intentaban aprender árabe. Los autores señalan que la motivación religiosa se compone de dos orientaciones: extrínsecas e intrínsecas. La conexión mediadora entre ambas es que el árabe es la lengua sagrada del Islam, y los investigadores lo afirman como parte del análisis de la motivación del alumno que estudia lenguas sagradas, en particular las personas que tienen una conexión significativa con la fe. La motivación espiritual debe tenerse en cuenta como un elemento independiente.

La noción de Dörnyei del yo futuro, la noción de Bakar et al. de la motivación religiosa, y el concepto de Joseph de la identidad religiosa sirven como una base útil en la cual se puede examinar las conexiones entre la visión L2, la identidad personal y la fe, con un enfoque especial en la interacción con la motivación L2. Un estudio realizado por Lepp-Kaethlet y Dörnyei (2012) da un enfoque más cualitativo del tema, echando un vistazo a las conversaciones y entrevistas formales con los alumnos cristianos L2. El sistema motivacional del yo que Dörnyei desarrolló fue utilizado para investigar la relación entre el aprendizaje de idiomas y la fe, ya que también se refiere a la conexión entre la adquisición de lenguas extranjeras y la visión religiosa. Para estos estudiantes de idiomas, encontraron evidencia de una visión $L 2$, así como una motivación religiosa que trabajaban juntas para crear un comportamiento de aprendizaje de idiomas con extraordinaria duración e intensidad. Su estudio se centró en participantes que demostraron tanto un grado anormalmente alto de motivación en el aprendizaje L2 como una excelente utilización del aprendizaje de lenguas en cuanto vehículo hacia el estudio o el uso de un texto sagrado. Se centraron en siete participantes elegidos de entre un grupo más grande que había completado las entrevistas en persona, con una duración de entre 45 y 75 minutos. Los participantes procedían de varias nacionalidades diferentes que ya habían aprendido entre uno y siete idiomas, además de su lengua materna, con el fin de obtener acceso a la Biblia. Todos ellos tenían niveles educativos postsecundarios que oscilaban entre tres y nueve años (Lepp-Kaethlet y Dörnyei, 2012). Se identificaron dos fases diferentes del proceso de la motivación: por un lado la creación de la visión y, por otro, vivir esa visión. Estas fases vinieron de las entrevistas en profundidad que los siete participantes tuvieron con los investigadores.

Si se va a diseñar una instrucción L2 que tiene acceso a estas motivaciones religiosas, entonces, hay que tener estas dos fases en cuenta. Es provechoso analizar cada uno de estos pasos en las partes que los componen.

La creación de la visión tiene que ver, en primer lugar, con descubrir por qué los participantes están aprendiendo la L2. Al analizar esto, es importante comenzar con el contexto religioso y sociocultural. El determinar la fe religiosa de un alumno L2 y su texto deseado es el primer paso importante. Los alumnos para los que los estudios religiosos son tan importantes como para considerarlos como parte adicional de su aprendizaje lingüístico, muestran un interés más profundo en aprender sobre su fe. Para los seguidores religiosos, el texto sagrado es la base para todo (es la fuente de todas sus creencias y tradiciones). Más allá de eso, es una fuente de apoyo emocional y bienestar psicológico, porque creen que es la revelación infalible de su tradición. Hay tres elementos comunes a los estudiantes de este nivel en su motivación: la visión espiritual, la visión L2 y el texto sagrado.

La visión espiritual viene de una incitación interna que los estudiantes generalmente interpretan como un mandato divino en sus vidas, para que puedan entrar en las vocaciones de servicio, por ejemplo, servir como un estudioso de la Biblia o traductor, o un misionero dentro de su fe. Esta visión incluye una perspectiva dinámica de las mismas, progresando hacia una aspiración de su propio futuro dentro de esa comunidad. La visión L2 es un elemento particular de esta visión espiritual; cumplir con el llamamiento divino significa tomar la L2 
como parte de su identidad; el no hacerlo significaría faltar a su visión inicial. Para muchas personas que quieren ser misioneros, llegar a ser versado en el idioma extranjero es uno de los pasos iniciales en la formación, lo que hace que la motivación sea más fuerte. Curiosamente, Lepp-Kaethlet y Dörnyei (2012) informaron que algunos de sus participantes tenían un autoyo L2 futuro como parte de su identidad desde una temprana edad. Incluso, antes de que algunos de los participantes conocieran el idioma que ellos tendrían que aprender en el marco de este nuevo futuro, esperaban llegar a hablar un idioma desconocido y que no estudiaron desde niños. Uno de los participantes informó a los investigadores que tenía sueños acerca de sí mismo en donde sabía una lengua extranjera y la usaba para trabajar con otros. Estos sueños procedían, en parte, de su "llamamiento divino", de tal modo que una vocación espiritual y ese autoyo L2 futuro de sus sueños se convirtió en una parte integral de sus propias aspiraciones. Esta conexión es una poderosa motivación para este entrevistado en particular; forjar la sinergia entre el aprendizaje de idiomas y el llamamiento puede motivar a muchos.

El texto sagrado es el tercer componente de la creación de la visión. En el caso del contexto cristiano, el aprendizaje de la lengua original implica un conocimiento de lectura del hebreo y el griego, si bien no es habitual que los que entran en el ministerio cristiano dominen la pronunciación y comprensión auditiva para estos dos idiomas. Esos son los dos idiomas más comunes estudiados por los seminaristas, al menos en lo que lectura e interpretación respecta. Para los estudios de doctorado, un lenguaje moderno también es requerido, y una opción común es el alemán, ya que los textos protestantes tempranos fueron escritos por Martín Lutero. En la tradición católica, el latín es una lengua que se enseña en un contexto L2 para la comprensión de la liturgia latina, así como los escritos originales o los padres de la Iglesia. El interés primordial en las lenguas modernas, sin embargo, proviene de los misioneros, que tienen que aprender los idiomas de otras culturas con el fin de servirlas. Los que participan en el aprendizaje L2, y para poder leer las len- guas originales de los textos sagrados, por supuesto, tienen más motivación que todos para acceder a la grabación original de la revelación divina.

El pasar de crear la visión a vivirla consiste en tomar la motivación original y convertirla en acción. Hasta el momento, se han analizado las razones por las que uno estudiaría un idioma L2 en relación con su religión, y se ha encontrado que existe una combinación de una vocación religiosa, una visión de sí mismos como seres L2 y un deseo de tener acceso, y facilitar el acceso de otros, a un texto sagrado. Dentro de la idea de vivir la visión, un criterio tiene que ver con la calidad y la intensidad de la conducta orientada a objetivos. Cuando la decisión a estudiar una lengua L2 tiene que ver con un sentido de vocación religiosa el tipo de vocación que requiere una interrupción importante en la vida también implica otros cambios. Desde el punto de vista de la persona que entra en el ministerio de la Iglesia, el compromiso implica la aceptación de una vida que, a menudo, tiene poca permanencia residencial, ya que los ministros se mudan frecuentemente. Para la persona que entre en el campo de la misión, el compromiso puede consistir en mudarse a otro país y vivir allí durante la mayor parte de su vida. El cambio de país puede implicar trasladarse a lugares donde la pobreza es extrema, la política es caótica, los climas son duros, hay pocos compañeros con quien hablar y la atención médica es deficiente. Los salarios propios pueden depender en gran medida de las donaciones de los feligreses. Los idiomas que se aprenden, dependiendo de dónde se encuentre el campo de misión, pueden estar fuera de los planes de estudio normales que uno encuentra en las universidades actuales. Mientras que el aprender una lengua extranjera en sí mismo puede parecer una tarea muy importante para muchos, para la persona que se dirige hacia el otro lado del globo a vivir, es solo un componente más del cambio que viene con el cumplimiento del llamamiento de Dios. Es lógico, entonces, que el tener ese tipo de motivación apasione a alguien a trabajar duro y mucho en el aprendizaje de la lengua de su nuevo mundo. Ser capaz de comprender el texto original de la Biblia y traducir tal iluminación a los idiomas de los demás sería una 
poderosa fuente de inspiración para los estudiantes L2 que deseen cambiar el mundo para los demás.

Otro criterio para vivir la visión tiene que ver con la duración del comportamiento orientado a los objetivos. Incluso, para las personas que entran en el campo de la misión a largo plazo, la conservación es una tarea difícil. Según los resultados de un informe de 2003 de EE. UU. sobre la retención misionera encargado por la Alianza Evangélica Mundial, la tasa media anual de retención total de todas las 559 agencias que participaron fue 94,56 por ciento, a lo largo de diez años, el 43 por ciento de los misioneros salen del campo, en promedio (World Evangelical Alliance, 2003). Eso significa que casi la mitad de los misioneros dejan el campo cada diez años; dado el idealismo con el que todos los misioneros se establecen, este es un número desalentador. Sin embargo, quienes tienen el compromiso de pasar años para dominar los idiomas locales de los campos de sumisión como parte de un llamamiento espiritual, tienen más probabilidades de tener éxito no solo en el aprendizaje de las lenguas extranjeras, sino también en los campos de misión en general.

\section{Implicaciones para el diseño curricular}

Hay varias implicaciones de esta investigación que vale la pena explorar. En primer lugar, parece que hay un grado intrínseco de la motivación para la adquisición de un idioma extranjero que se ajusta a las identidades de los propios alumnos. Para convertirse en su yo ideal, tienen que abrazar la fluidez L2 $y$, para eso, tienen que tener una motivación natural para perseguirlo y lograrlo. Merece la pena preguntarse qué implicaciones tiene esto para el aula L2. A fin de que más cursos de lenguas extranjeras se aprovechen de esta fuente oculta de la motivación, ¿qué es lo que debe suceder? Sirve de ayuda volver al tercer pilar de Dörnyei del sistema de automotivación, que tiene que ver con la experiencia de aprendizaje L2. Estos motivos no vienen del estudiante sino del ambiente de aprendizaje. El impacto del maestro, el clima de aula, el currículo y la experiencia global de éxito forman parte del aprendizaje. ¿Cuáles son algunas de las maneras en las que el impulso religioso puede ser utilizado para mantener el compromiso con la disciplina, parte importante del aprendizaje L2? ¿Cómo pueden los estudiantes ver el aprendizaje L2 como un uso agradable, significativo y beneficioso de su tiempo? Hay varios elementos que vale la pena considerar en esta área.

Debido a que el aprendizaje L2 está tan enlazado con la identidad del alumno, merece la pena examinar el contenido del plan de estudios para ver cómo se fomenta un cambio de autoidentificación para él. Smith y Carvill (2000) han señalado que el contenido curricular L2 encasilla a menudo la identidad del alumno y su propia imagen. El vocabulario y el contenido de los capítulos, por ejemplo, pueden animar al alumno a verse a sí mismo como una pieza más simple dentro de la maquinaria más grande de una economía, como consumidor, o como turista. Las unidades que contienen vocabulario de los alimentos, por ejemplo, se enseñan a menudo en el contexto de pedir algo en un restaurante o ir a la tienda de comestibles para hacer algunas compras. Aunque esto puede ser útil para ayudar al alumno L2 a hacerlo a través de sus primeras semanas en una nueva sociedad, Smith y Carvill (2000) argumentan que esta no es la manera más óptima educativamente - ni la más eficaz- de enseñar este contenido. En lugar de enseñar vocabulario sobre comida y bebida solo en el contexto de consumo, otro medio podría ser el de participar en las discusiones sobre las sociedades en las que no hay suficiente comida para todos. Otra observación que Smith y Carvill (2000) han hecho es que los programas L2 rara vez, si acaso, ahondan en preguntas sobre los significados más profundos de la vida o la identidad de la fe que el aprendiz de la lengua tiene. Es muy posible que todos los alumnos L2 dieran la bienvenida a un cambio que incluya algunas de estas cuestiones. Es casi seguro que las personas que han entrado en los estudios L2 con el fin de seguir una vocación religiosa estarían de acuerdo. Norton (2001) realizó un estudio sobre el caso de dos estudiantes canadienses L2 que consistía en largas entrevistas en persona. A la larga, se retiraron de sus clases de inglés, ya que no les parecían ser suficientemente estimulantes. Una de las causas de su retiro, sostiene Norton, es la falta 
de conexión entre la percepción de los estudiantes sobre su propio mundo, en oposición al mundo que la clase L2 les presenta. Ellos creían que su comunidad era más grande que las cuatro paredes que conforman el aula académica; sin embargo, el enfoque altamente pragmático de su plan de estudios L2 no tomó en cuenta los factores más grandes y globales y, como resultado, la clase no abordó la totalidad de sus necesidades educativas.

En el diseño de las actividades de instrucción en el aula, es importante incluir estos asuntos de una manera más profunda en las cuestiones de la existencia si uno está enseñando L2 u otra materia. Esto involucra los conceptos de comparaciones y conexiones de los estándares nacionales de ACTFL. Aparte, dentro de las aulas L2 en los primeros dos años de instrucción, hay varias formas de agregar elementos de los principios cristianos (o de otra religión) en la enseñanza. Por ejemplo, la unidad de alimentación y bebidas podría incluir una discusión sobre gente necesitada, así como una comparación entre lo que la gente come ahora en las diversas culturas en cuestión y lo que la gente comía en tiempos bíblicos. Para entablar el área de las culturas (de las 5 c), la unidad en el vocabulario del hogar podría incluir los elementos que un misionero utiliza cuando vive en un país de la lengua meta. Si un maestro nunca llega a hablar de estas áreas, entonces, el maestro no se ocupa de las áreas de la vida que más les importan a los estudiantes y, como resultado, la clase nunca hará conexiones con esas áreas cruciales y se desmotivará. La enseñanza L2 debe incluir la exploración de las preguntas más grandes en varias áreas, artículos periodísticos y columnas de opinión, fuentes L2 sobre temas sociales, así como artículos y columnas de opinión L1 sobre temas sociales en la cultura meta. Algunas cuestiones de importancia religiosa también deberían abordarse en las lecturas, tanto L1 como L2. Estas cuestiones son importantes, da igual el idioma de que se hable, y un currículo L2 completo lo reconocería.

Otra consecuencia a deducir de esto es que los textos sagrados pueden ser poderosos materiales curriculares, si se está estudiando en una institu- ción religiosa o persiguiendo objetivos religiosos. Las motivaciones del alumno pueden ser fortalecidas a través del uso de los textos sagrados. Por ejemplo, las universidades estatales en China usan la Biblia, a pesar de que las actividades evangélicas están prohibidas en el país. Debido a que los chinos ven la Biblia como una ventana importante a las costumbres de Occidente, incluyendo facultades de derecho, economía, literatura e historia, la Biblia (en inglés) es uno de los textos más utilizados en China para la enseñanza del inglés (Zetzsche, 1997). Esta es la razón de la proliferación de cuentos sobre la Biblia en las universidades chinas. Un área de investigación sería estudiar a los profesores, estudiantes y administradores educativos que fomentan el uso de los textos sagrados en el aula, a pesar de no compartir las creencias religiosas que se enseñan en esos textos, para saber por qué utilizan esos textos en el aula. Es probable que las razones que tengan aporten factores explicativos que se adhieren al movimiento "lengua a través de la literatura".

Los textos sagrados tienden a ocuparse de las cuestiones más profundas que los estudiantes quieren discutir, pero no se encuentran mucho en el aula. Además, los textos sagrados discuten sobre las principales cuestiones éticas y morales que son importantes para una cultura determinada, por lo que los textos pueden ser interesantes desde un punto de vista antropológico. En cualquier caso, el uso de los textos sagrados puede servir como un trampolín para la discusión significativa en clase, lo cual involucraría al alumno de una manera que solo un énfasis exclusivamente pragmático perdería. Los diseñadores curriculares deberían utilizar los textos sagrados para aumentar el nivel de pensamiento crítico que tiene lugar en el aula L2. Para activar los conceptos de comunicación, las actividades de clase L2 podrían incluir lecturas de la Biblia o cuentos infantiles religiosos en el idioma meta, en función del nivel lingüístico de los estudiantes. Discutir diferentes formas en que la fe religiosa es una parte de la vida en los distintos países meta llevaría a cabo el concepto de comunidades. 
Además, los profesores L2 pueden encuestar con frecuencia a sus estudiantes para conocer sus necesidades autoidentificadas y pueden diseñar planes individualizados de los materiales curriculares. Mientras las actividades de conversación son más propensas a ser para toda la clase, las lecturas y las respuestas escritas pueden basarse en textos distintos para los diferentes miembros de la clase. Los textos sagrados podrían animar a algunos alumnos más que a otros, y si la encuesta de autoidentificación indica que algunos (o todos) de los estudiantes se benefician de este tipo de actividad, pues, se los podría añadir a la mezcla curricular, lo cual favorecería un aprendizaje para todos.

En general, los resultados de esta investigación indican que hay una fuente potencialmente poderosa de motivación para los estudiantes que buscan la adquisición de una lengua extranjera, que ha estado en gran parte sin explorar dentro del campo más amplio de la lingüística aplicada, utilizando el sistema motivacional del yo en el análisis de la relación entre la visión L2 y la visión espiritual. Parece que el uso de textos sagrados puede ser una fuente de determinación y energía significativa para algunos que están llevando a cabo estudios L2. Cuando tres componentes (llamamiento divino, visión L2, y un texto sagrado) se utilizan en combinación, uno con el otro, y se canaliza de una manera significativa, el resultado final parece ser un flujo anormalmente alto de la motivación para el aprendizaje L2. Parece haber una fuerte fuerza interior, que bien puede ser una parte de las identidades intrínsecas de los alumnos, que los anima a aprender la L2 con un vigor inusual, persistencia y longevidad. La conclusión de estos resultados debería alentar a las personas que diseñan materiales educativos y planes de estudio y a los que enseñan las clases L2 a tratar de utilizar este tipo de fuentes para motivar a sus alumnos y traer mejores resultados al aprendizaje de idiomas. Incluso, si uno no está enseñando en un seminario o en un instituto de entrenamiento misionero, los beneficios del acceso a las partes de la inteligencia que tienen que ver con cuestiones más profundas (tanto de importancia social, así como de la importación existencial) tienen que ver con el ejercicio de la mente en niveles que no existiría en un plan de estudios más pragmático. Los beneficios adicionales que vienen con una mayor motivación y compromiso intelectual auguran un bien para los que tratan de mejorar la instrucción L2 para todos los estudiantes, independientemente de si se dirigen al extranjero para el campo misionero o, simplemente, si quieren ampliar su gama de oportunidades profesionales después de terminar su escolarización.

\section{Referencias}

American Council on the Teaching of Foreign Languages (ACTFL) (1996). Standards for Foreign Language Learning: Preparing for the $21^{\text {st }}$ Century.

Bakar, K. A., Sulaiman, N. F., y Rafaai, Z. (2010). Selfdetermination theory and motivational orientation of Arabic learners: A principal component analysis. GEMA Online Journal of Language Studies, 10(1), 71-86.

Coupland, N. (2010). The handbook of language and globalization. New York: John Wiley.

Crystal, D. (1965). Linguistics, language and religion. London: Burns Oats/Hawthorn Books.

Deci, E., y Ryan, M. (1985). Intrinsic motivation and self-determination in human behavior. New York: Plenum Press.

Dörnyei, Z. (2005). The psychology of the language learner: Individual differences in second language acquisition. Mahwah, NJ: Lawrence Erlbaum.

Dörnyei, Z. (2009). The L2 motivational self system. En Z. Dörnyei, E. Ushioda (Eds.), Motivation, language identity and the L2 self (pp. 9-42). Bristol, UK: Multilingual Matters.

Gilmore, A. (2007). Authentic materials and authenticity in foreign language learning. Language Teaching, 40, 97-118.

Joseph, J. E. (2004). Language and identity: National, ethnic, religious. Basingstroke, UK: Palgrave Macmillan.

Lepp-Kaethler, E., y Dörnyei, Z. (2012). The role of sacred texts in enhancing motivation and living the vision in second language acquisition. ELT and Christian Faith. New York: Routledge.

Maehr, M. L. (2005). The meaning that religion offers and the motivation that may result. Judeo-Christian perspectives on psychology: Human nature, motiva- 
tion, and change. Washington, DC: American Psychological Association.

Markus, H., y Nurius, P. (1986). Possible selves. American Psychologist, 41, 954-969.

McGrath, A. (2010). The passionate intellect: Christian faith and the discipleship of the mind. Downers Grove, IL: InterVarsity Press.

Miller, W. R., y Delaney H. (Eds.) (2005). Judeo-Christian perspectives on psychology: Human matter, motivation and change (pp. 133-144). Washington, DC: American Psychological Association.

Mooney, A. (2010). Has God gone global? Religion, language and globalization. The Handbook of Language and Globalization. Oxford, UK: Wiley-Blackwell.

Norton, B. (2001). Non-participation, imagined communities, and the language classroom. En M. Breen (Ed.), Learner contributions to language learning: New directions in research (pp. 159-171). Harlow, England: Pearson Education.

Smith, D., y Carvill, B. (2000). The gift of the stranger: Faith, hospitality and foreign language learning. Grand Rapids, MI: Eerdmans.

Thomas, S. (2005). The global resurgence of religion and the transformation of international relations: The struggle for the soul of the twenty-first century. New York: Palgrave Macmillan.

Wong, M. S. \& Canagarajah, A. S. (2009). Christian and critical English language educators in dialogue: Pedagogical and ethical dilemmas. New York: Routledge.

World Evangelical Alliance (2003). U.S. report of findings on missionary retention. http://www.worldevangelicals.org/

Zetzsche, J. O. (1997). Cultural primer or "Bible Stories" in contemporary mainland China. Asian and African Studies, 6(2), 217-232. 